\title{
COMPACT RIEMANNIAN MANIFOLDS WITH POSITIVE CURVATURE OPERATORS
}

\author{
BY JOHN DOUGLAS MOORE
}

The Riemann-Christoffel curvature tensor $R$ of a Riemannian manifold $M$ determines a curvature operator

$$
R: \Lambda^{2} T_{p} M \rightarrow \Lambda^{2} T_{p} M,
$$

where $\Lambda^{2} T_{p} M$ is the second exterior power of the tangent space $T_{p} M$ to $M$ at $p$, by the explicit formula

$$
\langle R(x \wedge y), z \wedge w\rangle=\langle R(x, y) w, z\rangle .
$$

$M$ is said to have positive curvature operators if the eigenvalues of $R$ are positive at each point $p \in M$. Meyer used the theory of harmonic forms to prove that a compact oriented $n$-dimensional Riemannian manifold with positive curvature operators must have the real homology of an $n$-dimensional sphere [GM, Proposition 2.9]. Using the theory of minimal two-spheres, we will outline a proof of the following stronger result.

THEOREM 1. Let $M$ be a compact simply connected $n$-dimensional Riemannian manifold with positive curvature operators, where $n \geq 4$. Then $M$ is homeomorphic to a sphere.

Theorem 1 is actually a consequence of another theorem which makes a weaker hypothesis on the curvature tensor. To describe this hypothesis, we extend the Riemannian metric $\langle$,$\rangle in two ways to the complexified tangent$ space $T_{p} M \otimes C$ : as a complex symmetric bilinear form $($,$) and as a Hermitian$ inner product $\langle\langle\rangle$,$\rangle . Similarly, we extend the metric in two ways to \Lambda^{2} T_{p} M \otimes$ C. An element $z \in T_{p} M \otimes \mathbf{C}$ is said to be isotropic if $(z, z)=0$. A complex linear subspace $V \subseteq T_{p} M \otimes \mathbf{C}$ is totally isotropic if $z \in V \Rightarrow(z, z)=0$.

Finally, we extend the curvature operator $R$ to a complex linear map $R: \Lambda^{2} T_{p} M \otimes \mathbf{C} \rightarrow \Lambda^{2} T_{p} M \otimes \mathbf{C}$.

DEFINITION. The curvature operator $R$ is positive on complex totally isotropic two-planes if whenever $\{z, w\}$ is a basis for a totally isotropic subspace of $T_{p} M \otimes \mathbf{C}$ of complex dimension two,

$$
\langle\langle R(z \wedge w), z \wedge w\rangle\rangle>0 .
$$

(Note that $M$ has positive sectional curvatures if and only if its curvature operator $R$ is positive on real two-planes.)

By means of a purely algebraic argument, it is possible to prove that if the sectional curvatures $K(\sigma)$ of a Riemannian manifold $M$ of $\operatorname{dim} \geq 4$ satisfy the inequality $1 / 4<K(\sigma) \leq 1$, then the curvature operator of $M$ is positive

Received by the editors November 1, 1985.

1980 Mathematics Subject Classification. Primary 53C20.

(C) 1986 American Mathematical Society $0273-0979 / 86 \$ 1.00+\$ .25$ per page 
on complex totally isotropic two-planes. Hence the following theorem implies not only Theorem 1, but also the Rauch-Berger-Klingenberg sphere theorem for manifolds of $\operatorname{dim} \geq 4$ [GKM, §7.8].

THEOREM 2. Let $M$ be a compact simply connected $n$-dimensional Riemannian manifold whose curvature operator is positive on complex totally isotropic two-planes, where $n \geq 4$. Then $M$ is homeomorphic to a sphere.

SKeTCH OF PROOF OF THEOREM 2. Let $S^{2}=\mathbf{C} \cup\{\infty\}$ be the Riemann sphere with the standard complex coordinate $z=x+i y$. If $f: S^{2} \rightarrow M$ is a conformal branched minimal immersion, and $V$ is a $C^{\infty}$ section of $f^{*} T M$, then the second derivative of the energy in the direction of the variation field $V$ is given by the index form

$$
\begin{aligned}
I(V, V)= & \int_{S^{2}}\left\{\left|\nabla_{\partial / \partial x} V\right|^{2}+\left|\nabla_{\partial / \partial y} V\right|^{2}\right. \\
& -\langle R(V, \partial f / \partial x) \partial f / \partial x, V\rangle-\langle R(V, \partial f / \partial y) \partial f / \partial y, V\rangle\} d x \wedge d y .
\end{aligned}
$$

It is convenient to extend this index form to a Hermitian symmetric form on $C^{\infty}$ sections of $f^{*} T M \otimes \mathbf{C}$. If $W=U+i V$, where $U$ and $V$ are smooth sections of $f^{*} T M$, then integration by parts (as in [M]) yields the formula (2)

$$
\begin{aligned}
I(W, W) & =I(U, U)+I(V, V) \\
& =4 \int_{S^{2}}\left\{\left\|\nabla_{\partial / \partial \bar{z}} W\right\|^{2}-\langle\langle R(W \wedge \partial f / \partial z), W \wedge \partial f / \partial z\rangle\rangle\right\} d x \wedge d y .
\end{aligned}
$$

In this formula, $\partial f / \partial z$ is the section of $f^{*} T M \otimes \mathbf{C}$ defined by

$$
(\partial f / \partial z)(p)=(1 / 2)\left(f_{*_{p}}\left(\partial /\left.\partial x\right|_{p}\right)-i f_{*_{p}}\left(\partial /\left.\partial y\right|_{p}\right)\right), \quad \text { for } p \in S^{2} \text {. }
$$

$f^{*} T M \otimes \mathbf{C}$ can be made into a holomorphic vector bundle over $S^{2}$ in a unique fashion so that the local holomorphic sections of $f^{*} T M \otimes \mathbf{C}$ are exactly the sections annihilated by $\nabla_{\partial / \partial \bar{z}}$. When this is done, the fact that $f$ is conformal and harmonic implies that $\partial f / \partial z$ is an isotropic holomorphic section of $f^{*} T M \otimes \mathbf{C}$. A theorem of Grothendieck $[\mathbf{G}]$ implies that $f^{*} T M \otimes \mathbf{C}$ can be decomposed into a direct sum of holomorphic line bundles, $f^{*} T M \otimes$ $\mathbf{C}=L_{1} \oplus L_{2} \oplus \cdots \oplus L_{n}$, where

$$
c_{1}\left(L_{1}\right) \geq c_{1}\left(L_{2}\right) \geq \cdots \geq c_{1}\left(L_{n}\right), \quad c_{1}\left(L_{n-i}\right)=-c_{1}\left(L_{i}\right) .
$$

(Here $c_{1}\left(L_{i}\right)$ denotes the first Chern class of $L_{i}$ evaluated on the fundamental cycle of $S^{2}$.) This direct sum decomposition allows us to give a lower bound on the dimension of the space of isotropic holomorphic sections of $f^{*} T M \otimes \mathbf{C}$, and this bound, together with formula (2), can be used to establish

PROPOSITION. If $f: S^{2} \rightarrow M$ is a nonconstant conformal branched minimal immersion into a Riemannian manifold whose curvature operator is positive on complex totally isotropic two-planes, then the index form (1) at $f$ has index $\geq(n / 2)-(3 / 2)$.

(By the index of a symmetric bilinear form, we mean the dimension of a maximal linear subspace of the domain on which the form is negative definite.) 
Now we utilize the $\alpha$-energy of Sacks and Uhlenbeck [SU], the real-valued $C^{2}$ function on the Banach manifold $L_{1}^{2 \alpha}\left(S^{2}, M\right)$, where $\alpha$ is slightly greater than 1 , defined by

$$
E_{\alpha}(f)=\int_{S^{2}}\left(1+|d f|^{2}\right)^{\alpha} d \mu .
$$

Here $S^{2}$ is given the metric of constant curvature having volume one, $d \mu$ is the area element with respect to this metric, and $|d f|^{2}$ is the energy density. We regard $M$ as isometrically imbedded in an ambient Euclidean space $E^{N}$, and set

$$
T_{f} L_{1}^{2 \alpha}\left(S^{2}, M\right)=\left\{V: S^{2} \rightarrow E^{N} \mid V(p) \in T_{f(p)} M, \quad \text { for all } p \in S^{2}\right\} .
$$

To any critical point $f$ for $E_{\alpha}$ is associated its Hessian, a continuous symmetric bilinear form

$$
d^{2} E_{\alpha}(f): T_{f} L_{1}^{2 \alpha}\left(S^{2}, M\right) \times T_{f} L_{1}^{2 \alpha}\left(S^{2}, M\right) \rightarrow \mathbf{R} .
$$

LEMMA. Let $k$ be the least integer, $2 \leq k \leq n$, such that $\pi_{k}(M) \neq 0$. Then there is a nonconstant critical point $f$ for $E_{\alpha}$ such that the Hessian of $E_{\alpha}$ at $f$ has index $\leq k-2$.

Indeed, if $E_{\alpha}$ did not have any nonconstant critical points of index $\leq k-2$, it could be approximated by a function whose nonconstant critical points were weakly nondegenerate (in the sense of Uhlenbeck [ $U$, p. 432]) and of index $\geq k-1$. Then Morse theory on Banach manifolds $[\mathbf{U}, \mathbf{T}]$ would imply vanishing of the relative homotopy group

$$
\pi_{k-2}\left(L_{1}^{2 \alpha}\left(S^{2}, M\right), \hat{M}\right)=0,
$$

where $\hat{M}$ is the subspace of constant maps from $S^{2}$ to $M$. This would contradict $\pi_{k}(M) \neq 0$.

By the lemma, we can choose a sequence of nonconstant critical points $f_{\alpha(i)}$ for $E_{\alpha(i)}$ of index $\leq k-2$, with $\alpha(i) \downarrow 1$. By [SU], we can assume that $E_{\alpha}\left(f_{\alpha}\right) \leq\left(1+B^{2}\right)^{\alpha}$ and energy $\left(f_{\alpha}\right) \geq \varepsilon$, where $B$ and $\varepsilon$ are positive constants independent of $\alpha$. After passing to a subsequence, we can arrange that the $f_{\alpha(i)}$ 's will $C^{1}$-converge on $S^{2}$ minus a finite number of points to a conformal branched minimal sphere [SU, Theorem 4.4]. If the limiting sphere is nonconstant, it can be shown that its index form has index $\leq k-2$. (We can neglect the finite number of points at which convergence fails by an argument of Gulliver and Lawson [GL, Proposition 1.9].)

If the limiting sphere is constant, then a nontrivial branched conformal minimal sphere must "bubble off" as $\alpha \rightarrow 1$ [SU, Theorem 4.6]. In this case, a nontrivial bubbled-off sphere must have index $\leq k-2$.

The proposition now implies that $k-2 \geq(n / 2)-(3 / 2)$. Hence $\pi_{i}(M)=0$, for $1 \leq i \leq n / 2$. It thus follows from Poincaré duality that $M$ is a homotopy sphere, and by the resolutions of the generalized Poincaré conjecture when $n \geq 4, M$ must be homeomorphic to a sphere.

More details will appear in a subsequent article.

ADDED IN PROOF. The author has recently been informed that Micallef has independently obtained results similar to Theorems 1 and 2 . 


\section{REFERENCES}

[GM] S. Gallot and D. Meyer, Opérateur de courbure et Laplacien des formes différentielles d'une variété riemannienne, J. Math. Pure Appl. 54 (1975), 259-284.

[GKM] D. Gromoll, W. Klingenberg and W. Meyer, Riemannsche Geometrie im Grossen, Lecture Notes in Math., vol. 55, Springer-Verlag, Berlin, 1968.

[G] A. Grothendieck, Sur la classification des fibres holomorphes sur la sphère de Riemann, Amer. J. Math. 79 (1957), 121-138.

[GL] R. Gulliver and H. B. Lawson, The structure of stable minimal hypersurfaces near a singularity (to appear).

$[\mathbf{M}]$ J. D. Moore, On stability of minimal spheres and a two-dimensional version of Synge's theorem, Archiv der Math. 44 (1985), 278-281.

[SU] J. Sacks and K. Uhlenbeck, The existence of minimal immersions of 2-spheres, Ann. of Math. (2) 113 (1981), 1-24.

[T] A. Tromba, A general approach to Morse theory, J. Differential Geom. 12 (1977), $47-85$.

[U] K. Uhlenbeck, Morse theory on Banach manifolds, J. Funct. Anal. 10 (1972), 430-445.

Department of Mathematics, University of California, Santa BarBARA, CALIFORNIA 93106 